\title{
A Produção Mais Limpa no Âmbito da Cadeia de Suprimentos Verde: Uma Análise no Setor de Panificação
}

A Cleaner Production Under the Green Supply Chain : An Analysis in the Bakery Industry

Producción Más Limpia Bajo la Cadena de Suministro Verde: Un Análisis en la Industria Panadera

Éllen Fernanda Gomes de Souza Mestranda, UNINOVE, Brasil. ellenfernandag@hotmail.com

Heidy Rodriguez Ramos Professora Doutora, UNINOVE, Brasil. heidyrr@uninove.br

Mauro Silva Ruiz Professor Doutor, UNINOVE, Brasil. maurosilvaruiz@uninove.br 


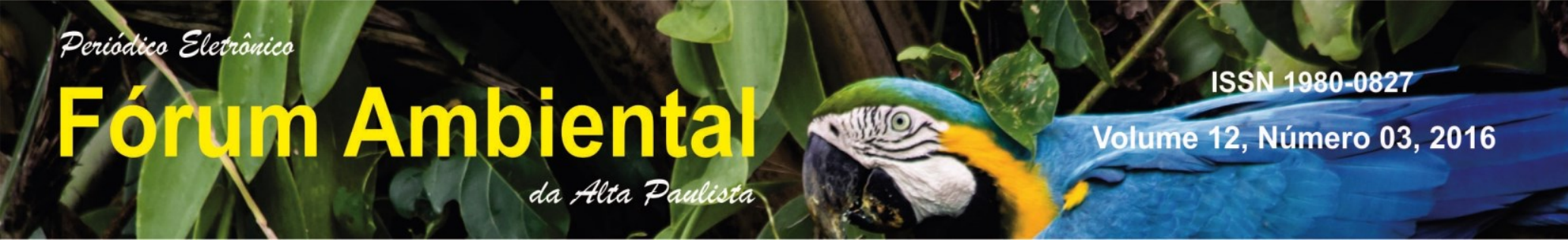

RESUMO

O objetivo geral é apresentar as melhorias que a produção mais limpa ocasionou ao setor de panificação, fundamentado na cadeia de suprimento baseado em resultados de estudos realizados. 0 presente estudo constituiu em uma pesquisa qualitativa de revisão bibliográfica baseada nos assuntos abordados de cadeia de suprimento verde, produção mais limpa, o setor de panificação e os resultados trazidos para as panificadoras. Foram apresentados resultados de várias pesquisas, com o intuito de apresentar os benefícios que a aplicação ambiental e de produção mais limpa dentro da cadeia de suprimento, trouxeram para as panificadoras. Nesse estudo foi possível observar que as técnicas de redução de resíduos, minimização de impacto ambiental, de poluição, descarte e redução de compras, têm sido uma das principais formas ambientais aliadas às indústrias. As operações verdes fazem parte no envolvimento da gestão da cadeia de suprimentos verde e as panificadoras não tem ficado fora dessas mudanças. Alguns pesquisadores têm ressaltado a proporção da importância que as panificadoras têm trazido ao Brasil, e nesse estudo são abordadas as melhorias trazidas dentro da cadeia de suprimento com subsídio da produção mais limpa.

PALAVRAS-CHAVE: Cadeia de suprimentos verde. Produção mais limpa. Panificação.

\section{ABSTRACT}

The overall goal is to present the improvements that cleaner production led to the bakery sector, grounded on the supply chain based on results of studies. This study consisted of a qualitative study of literature review based on the topics covered in green supply chain, cleaner production, the baking industry and the results brought to the bakeries. The results were presented in various surveys, in order to present the benefits that environmental and application of cleaner production within the supply chain, brought to bakeries. In this study it was observed that waste reduction techniques, minimizing environmental impact, pollution, disposal and reduction of purchases, has been a major environmental forms allied industries. Green operations are part of the involvement of the management of green supply chain and bakeries have not been out of these changes. Some researchers have pointed out the proportion of the importance that bakeries have brought to Brazil, and this study addresses the improvements brought in the supply chain with benefits of cleaner production.

KEYWORDS: Green supply chain. Cleaner production. Baking.

\section{RESUMEN}

El objetivo general es el de presentar las mejoras que la producción más limpia llevó a la sector de la panadería, basado en la cadena de suministro sobre la base de resultados de estudios. Este estudio consistió en un estudio cualitativo de la revisión de la literatura sobre la base de los temas tratados en la cadena de suministro verde, producción más limpia, la industria de panadería y los resultados presentados a las panaderías. Los resultados se presentaron en varios estudios, con el fin de presentar los beneficios que el medio ambiente y la aplicación de producción más limpia en la cadena de suministro, traído a las panaderías. En este estudio se observó que las técnicas de reducción de residuos, minimizando el impacto ambiental, la contaminación, la eliminación y la reducción de las compras, han sido una de las principales formas ambientales de las industrias conexas. operaciones verdes son parte de la participación de la gestión de la cadena de suministro verde y panaderías no han estado fuera de estos cambios. Algunos investigadores han señalado la proporción de la importancia que las panaderías han traído a Brasil, y este estudio se dirige a las mejoras introducidas en los beneficios de la cadena de suministro de producción más limpia.

PALABRAS CLAVE: cadena de suministro verde; producción más limpia; hornear. 


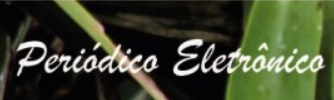

\section{INTRODUÇÃO}

Nas últimas décadas, empresas de diferentes ramos começaram a dar ênfase aos seus investimentos socioambientais de forma padronizada e profissional. A busca constante das organizações por ferramentas, métodos e técnicas que aplicadas ajudem a solucionar ou melhorar a preservação dos recursos naturais e a minimizar a degradação ambiental, vem levando as organizações a aliar estratégias para melhorar seu desenvolvimento organizacional.

De acordo com Henriques e Catarino (2014) no passado, as empresas olhavam para o meio ambiente como um grande problema, com custos e fatores de riscos. Porém, atualmente os empresários começaram a observar como fonte de melhoria, oportunidades, eficiência e crescimento. Um exemplo é a energia poupada, que diminui os custos de produção e as saídas indesejáveis, tais como os resíduos que são encurtados.

A cadeia de suprimentos verde interliga toda a cadeia produtiva de uma organização, relacionando toda a produção a uma mesma finalidade para criar aspectos sustentáveis em todo o processo, desde a entrada das mercadorias até o produto final a ser entregue ao consumidor. Apesar dos esforços para aplicar ferramentas ambientais, a cadeia de suprimentos verde trás alguns subsídios para ajudar a beneficiar as partes envolvidas da cadeia, a produção mais limpa é considera uma dessas ferramentas. A aplicação dessa ferramenta ambiental e de seu gerenciamento elimina o desperdício, contribuindo para melhoria ambiental.

Segundo a Centro Nacional de Tecnologias Limpas (CNTL), por meio da produção mais limpa é oportuno analisar a forma como um processo de produção está sendo concretizada e observar em quais etapas do processo as matérias-primas e os recursos estão sendo perdidos. Dessa forma, é possível reaproveitar, diminuir ou impedir a geração de resíduos. Para que a forma de produzir seja limpa, sendo, uma ação econômica e lucrativa, uma ferramenta importante para conservar adequadamente a legislação ambiental e ainda gerar o desenvolvimento sustentável (CNTL, 2005).

A questão da implementação da produção mais limpa, nas pequenas e médias empresas, demanda modificação no comportamento do sistema produtivo por meio de ajustes de uma postura pró-ativa dos empresários, e uma maior exigência por parte dos consumidores. (Rossi \& Barata, 2009).

O desenvolvimento acerca dos níveis de implantação, conscientização e organização de práticas ambientais vem sendo cobrada pela sociedade. Dessa forma, o segmento escolhido para estudo foi o de panificação. De acordo com a Associação Brasileira da Indústria de Panificação e Confeitaria (ABIP) esse segmento hoje representa um dos seis maiores segmentos indústrias no País. (ABIP, 2012). 


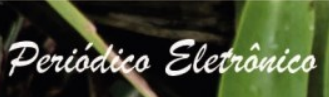

Com o embasamento dessas premissas, no que se relaciona ao problema da pesquisa aqui proposto, ele é exposto na forma da seguinte pergunta: Quais fatores a produção mais limpa pode acarretar como melhoria para a cadeia de suprimento de uma indústria de panificação?

O objetivo geral é apresentar as melhorias que a produção mais limpa ocasionou ao setor de panificação, fundamentado na cadeia de suprimento, baseado em resultados de estudos realizados por meio de uma revisão bibliográfica.

\section{REVISÃO BIBLIOGRÁFICA}

\subsection{CADEIA DE SUPRIMENTO VERDE}

Para Fernandes e Espinosa (2010) a cadeia de suprimento é um conceito de fluxo ampliado que integra desde os processos logísticos, incluindo as atividades envolvidas no fornecimento de insumos e matéria-prima, a transformação dos insumos e matéria-prima em novos produtos, até a distribuição dos produtos finais aos consumidores. Esse processo deve ser alinhado com a estratégia da empresa. Essas características influenciam a adotar práticas ambientais, desde o tamanho da instalação industrial até o processo produtivo.

Seuring e Muller (2008) definiram gestão da cadeia de fornecimento sustentável como a gestão de material, informação e fluxos de capitais, e a cooperação entre as compras ao longo da cadeia de abastecimento, tendo as três dimensões envolvidas do desenvolvimento sustentável, sendo derivados de requisitos dos clientes e partes interessadas.

Seuring e Muller (2008) completam que na cadeia de suprimento verde critérios ambientais e sociais precisam ser cumpridos pelos membros para permanecer dentro da cadeia de suprimento, enquanto a competitividade seria mantida por meio de atender às necessidades dos clientes e dos critérios econômicos relacionados.

Segundo Dias, Labegalini e Csillag (2012) aliar o papel social e ambiental para criar importância na cadeia de suprimentos é um desafio para os gestores desse século. Sendo inadequado distinguir as dimensões do desenvolvimento sustentável, ambiental, social e econômico, pois elas influenciam todas as organizações que constituem uma cadeia produtiva.

A gestão da cadeia de suprimento verde de acordo com Srivastava (2007) pode reduzir os impactos ecológicos das atividades industriais, sem sacrificar a qualidade, custo, confiabilidade, desempenho ou a utilização da eficiência energética. Trata-se de uma mudança de paradigma, para minimizar danos ecológicos e também levando a um lucro econômico global.

No estudo realizado as operações verdes foram delimitadas, envolvendo todos os aspectos operacionais relacionados ao que mais se aproxima do objetivo da produção mais limpa que é: gestão de resíduos envolvendo a disposição, a redução na fonte e a prevenção da poluição, conforme apresentado na figura 1. 
Figura 1 - Classificação da cadeia de abastecimento, a partir das operações verdes.

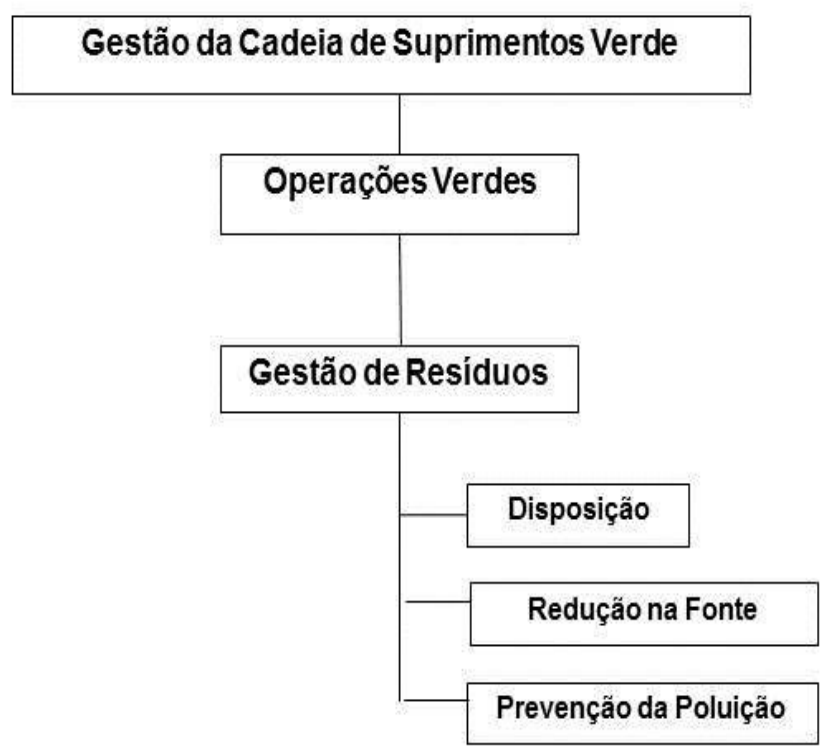

Fonte: Elaborado pelos autores a partir de Srivastava (2007)

Quando a gestão dos resíduos é realizada Glavic e Lukman (2007) descrevem que isso faz parte do desempenho industrial, referindo-se as dimensões de segurança e saúde humana. Reduzindo assim, custos e riscos para as organizações.

\subsection{PRODUÇÃO MAIS LIMPA}

A produção mais limpa surgiu em 1991, durante um Programa das Nações Unidas para o Meio Ambiente (PNUMA) (em inglês, United Nations Environmental Program - UNEP). (Petter, Vaz, Resende, Selig 2011; Barbieri 2004; Dias 2008).

O conceito de produção mais limpa menciona à produção integrada e a proteção ambiental de forma mais abrangente, equiparando as fases do processo produtivo e o ciclo de vida do produto final. (Petter et.al. 2011).

Filho e Sicsú (2003) descrevem a produção mais limpa, como uma estratégia aplicada à gestão ambiental. Sendo sugerida como uma ferramenta que permite o funcionamento da empresa de modo social e ambientalmente responsável, acarretando também em melhorias econômicas e tecnológicas.

Para Rossi e Barata (2009) a necessidade de pôr em atuação a ideia de ecoeficiência fez com que aparecesse a produção mais limpa. Com a implantação de técnicas de produção mais limpa, obtém redução do consumo de recursos, de impacto na natureza e condicionantes que incentivam a inovação e transparência. 
De acordo com o Centro Nacional de Tecnologia Limpa (CNTL), produção mais limpa, compõe o aproveitamento consecutivo de uma estratégia econômica, ambiental e tecnológica integrada aos processos e produtos, para aumentar a eficácia no uso de matérias-primas, água e energia por meio da não geração, diminuição ou reciclagem de resíduos provocados em todos os setores produtivos. (Petter et.al. 2011; Barbieri 2004).

Barbieri (2004) afirma que a produção mais limpa é considerada uma estratégia ambiental preventiva aplicada a processos, produtos e serviços para diminuir o impacto no meio ambiente.

Segundo Dias (2008) de acordo com o PNUMA, o programa para a produção mais limpa procura: aumentar o consenso mundial para uma visão de produção mais limpa; apoiar a rede de organizações dedicadas à promoção de estratégias de produção mais limpa e a ecoeficiência; ampliar as possibilidades de melhoria ambiental das empresas mediante a capacitação e a educação; apoiar projetos que sirvam de modelo de referência; fornecer assistência técnica.

De acordo com Baas (1995) a PNUMA em 1992 declarou quatro adicionais para responder a pergunta "o que é produção mais limpa"?:

(a) Produção mais limpa Significa a aplicação contínua de uma estratégia ambiental integrada e preventiva para ambos os processos e produtos, para reduzir riscos aos seres humanos e ao meio ambiente.

(b) As técnicas de produção mais limpa incluem a conservação de matérias-primas e energia, eliminando material tóxico dos processos e a redução da quantidade e toxicidade de todas as emissões e resíduos.

(c) A estratégia de produção mais limpa para produtos foca na redução dos impactos ambientais ao longo de todo o ciclo de vida do produto.

(d) A produção mais limpa é obtida pela aplicação de perícia, de melhoria tecnológica e mudanças de atitudes.

\section{O SETOR DE PANIFICAÇÃO NO BRASIL}

De acordo com a ABIP (2013) em 2012, o índice de crescimento das empresas de panificação e confeitaria no Brasil foi de $11,6 \%$, o que representa um faturamento de $\mathrm{R} \$ 70,29$ bilhões. $O$ setor de panificação vem contribuindo consideravelmente, nos últimos anos, para o crescimento do food service no Brasil. Estima-se que a participação da panificação foi em $36 \%$ do faturamento do setor de food service..

Segundo a ABIP (2012) a panificação no Brasil representa, um dos seis maiores segmentos industriais do país, sua participação no setor de produtos alimentícios é de 36,2\% e na indústria de transformação esse percentual é de $7 \%$. 


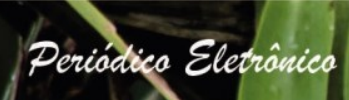

O número de panificadoras que compõem o setor permanece na casa dos $60 \mathrm{mil}$, e receberam cerca de 44 milhões de clientes no ano de 2011. O setor representa cerca de 802 mil empregos diretos e 1,85 milhão de forma indireta.

A ABIP (2013) destacou o perfil do setor de panificação no Brasil, abordando os seguintes tópicos:

- $\quad$ Em 2012, as vendas de produção própria representam 54,5\%, sendo a maior parte do volume de faturamento e atinge $\mathrm{R} \$ 39,2$ bilhões;

- $\quad$ O volume de faturamento abarca, inclusive, os cerca de $20 \%$ de empresas informais que compõem o setor;

- $\quad 23$ mil funcionários contratados pelas padarias em 2012.

\section{METODOLOGIA}

Martins e Theóphilo (2009, p.141) descreveram como um exemplo de dados qualitativos "citações diretas de pessoas sobre suas experiências". O presente estudo constituiu em uma pesquisa qualitativa, como objetivo o estudo foi exploratório, pois visa conhecer os atributos de um fenômeno, entendendo suas causas e consequências.

Quanto aos assuntos abordados de cadeia de suprimento verde e produção mais limpa e os resultados trazidos para as panificadoras constituíram em uma pesquisa bibliográfica. (Martins \& Theóphilo 2009; Vergara 2006). Foi realizado um estudo bibliográfico com o intuito de se aperfeiçoar no conhecimento, e na forma de se explicar contribuições à pesquisa em relação aos temas tratados, usando literaturas científicas.

Esse estudo bibliográfico foi realizado a partir das bases de dados on-line: Scopus e google acadêmico, utilizando as seguintes descrições em português: padarias, panificação, produção mais limpa e cadeia de suprimento. Em inglês foram descritos: bakeries, bakery, cleaner production e supply chain.

A revisão foi ampliada por meio de outras fontes, tais como cartilha de produção mais limpa de confeitaria e padarias do SENAI (2008) e arquivos da internet do site da Associação Brasileira de Indústria de Panificação - ABIP (2012) e o Programa de Desenvolvimento da Alimentação, Confeitaria e Panificação - PROPAN (2011), além de dissertações, livros, periódicos e artigos científicos. Foram apresentados resultados de várias pesquisas, com o intuito de expor os resultados que a aplicação ambiental dentro da cadeia de suprimento, apresentaram. No estudo foi abordado a prática da produção mais limpa como ferramenta de benefícios ao processo produtivo das panificadoras. (Martins \& Theóphilo 2009; Vergara 2006). 


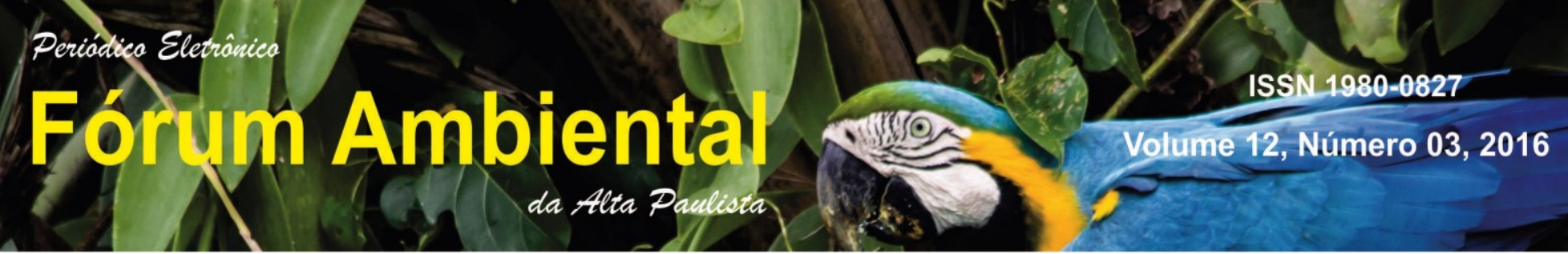

\section{RESULTADOS E DISCUSSÃO}

No presente estudo foi possível observar que a produção mais limpa é uma ferramenta que requer algumas mudanças na organização. As técnicas de redução de resíduos, minimização de impacto ambiental, de poluição, descarte e redução de compras, têm sido uma das principais formas ambientais aliadas as indústrias, que se encontra na ideia das operações verdes de Srivastava (2007) e as panificadoras não ficaram fora dessas mudanças.

Alguns pesquisadores têm ressaltado a importância das panificadoras no Brasil, e nesse estudo são abordadas as melhorias trazidas nesse processo, dentro da cadeia de suprimentos com subsídio da produção mais limpa.

Fernandes e Espinosa (2010) descrevem que os processos de produção mais limpa trazem oportunidade para que a cadeia de suprimento seja gerenciada de forma sustentável e que haja colaboração entre todos os participantes dessa cadeia. O sistema de produção é ecologicamente correto, com baixo custo da matéria-prima, água e energia elétrica. Esses aspectos estão nas condições de produção mais limpa.

De acordo com Glavic e Lukman (2007) produção mais limpa inclui tanto uma condição para obtenção de melhorias ambientais no processo e desenvolvimento de produtos, quanto para um mundo mais sustentável.

Fernandes e Espinosa (2010) descrevem que a melhoria da qualidade ambiental é seguida pelo desenvolvimento de tecnologias sustentáveis e pelas técnicas de produção e de gestão do setor industrial. E a metodologia de produção mais limpa, surge nesse contexto como um importante instrumento para diminuir efeitos que prejudicam o meio ambiente.

Jerônimo, Moura e Dantas (2013) percebem em seu estudo de um diagnóstico ambiental feito em padarias da cidade de Mossoró-RN, que esse segmento necessita de um maior rigor no cumprimento dos requisitos legais, e na regularização de alguns aspectos como o gerenciamento de resíduos, práticas de redução deles e a maximização do uso de recursos naturais e elementos finitos de gestão, pois assim podem garantir um melhor desempenho ambiental.

Na pesquisa de Fernandes e Freitas (2014) os autores analisaram um processo produtivo de uma panificadora de Campina Grande - Paraíba. Com a técnica de produção mais limpa foi observado que a panificação é um segmento industrial que vem se tornado muito importante na atividade econômica, contribuindo para o crescimento do país, demonstrado ser um causador de problemas ambientais. Esses problemas são basicamente relacionados com a eficiência produtiva levando a um maior consumo de matérias-primas e energia, e a geração de desperdícios e resíduos que são causadores de impactos ambientais negativos com custos consideráveis para qualquer empresa. Foram encontradas algumas limitações em relação a práticas ambientais. Já as oportunidades identificadas foram à padronização dos insumos e mudanças de matéria-prima, diminuição de resíduos sólidos e da energia. 
Pimenta e Gouvinhas (2007) propuseram em sua pesquisa, implementar um programa de produção mais limpa em uma pequena empresa no setor de panificação de Natal - RN. Os resultados obtidos foram redução de custos na fabricação do pão francês, redução do lead time, movimentação e inspeções, o uso eficiente de água, materiais e energia, menor geração de resíduos sólidos e o envolvimento da incorporação para condutas ambientais.

Os indicadores ambientais são um dos modelos que podem ser aplicados às organizações que implantaram nas indústrias o uso da produção mais limpa, esses indicadores podem ser disponíveis aos consumidores em forma de relatórios ambientais, trazendo benefícios à empresa e um maior relacionamento com a sociedade.

Cardoso (2004) descreve que a maior prova para as organizações é incluir em seu padrão a adoção da produção ambientalmente sustentável não em benefício de ser uma intenção no contexto empresarial ou um diferencial competitivo para os concorrentes, mas sim um compromisso de responsabilidade para o meio ambiente que necessita da ajuda de todos. É coerente implantar e apresentar uma proposta de indicadores de produção mais limpa, para serem utilizados em relatórios ambientais, porque os indicadores propõem um maior entendimento e comunicação, permitindo a sociedade seguir os processos ambientais das empresas.

A produção mais limpa tem como um das principais finalidades a minimização dos resíduos sólidos. Diante disso o estudo de Pimenta e Junior (2006) apresentou um modelo de gerenciamento dos resíduos sólidos para indústrias de panificação. O estudo de caso foi aplicado a uma padaria de Natal - RN, que mostrou que essa atividade apresenta uma potencialidade poluidora, no que se refere aos resíduos sólidos que geram e as técnicas de gerenciamento utilizadas. $O$ trabalho apresentou a criação de um modelo que envolveu várias etapas que contemplava desde a caracterização dos resíduos até o plano de gerenciamento. Para que os resíduos fossem destinados ao local correto. O modelo envolveu todos os funcionários da padaria e com a receita arrecadada dos resíduos recicláveis vendidos, criou-se um "fundo social" para os funcionários.

Pode-se observar que a produção mais limpa quando inseridas as produções industriais trazem diversos contextos de mudanças ao meio ambiente.

Filho e Sicsú (2003) relacionam que a produção mais limpa fixar-se no contexto como uma alternativa viável no duelo dos problemas ambientais das empresas (independente do segmento de atuação). Mesmo existindo algumas barreiras, não é difícil sua implantação nas empresas. E se pode constatar na prática que empresas nacionais que têm sido dianteira na implementação da produção mais limpa puderam demonstrar as vantagens competitivas geradas pela estratégia limpa de produção.

A produção mais limpa ajuda as empresas a ressaltarem o quanto é importante ter uma boa imagem diante da sociedade por meio de ações de preservação ambiental, e de processos e produtos, associando esses aspectos como a redução de custos e a melhoria do seu desempenho ambiental. (Hinz, Valentina \& Franco, 2006). 
Outra forma de trazer benefícios com a implantação da produção mais limpa são as boas práticas operacionais viabilizando novos investimentos na empresa. Exemplos de boas práticas da produção mais limpa são: a dosagem e mudanças na concentração de produtos; máxima utilização da capacidade do processo produtivo; reorganização do tempo de limpeza; eliminação de perdas; melhoria de logística; elaboração de manuais de boas práticas, treinamento e capacitação do pessoal envolvido no programa; alteração do fluxo de materiais e ajuste do layout; melhoria do sistema de informação; padronização de operações e procedimentos e substituição de matéria-prima no processo. (SENAI, 2008).

Filho e Sicsú (2003) descreveram que as boas práticas de fabricação constituem procedimentos administrativos e técnicos que permitem a minimização da produção de resíduos. Reduzindo a formação de resíduos ou o impacto ambiental negativo sob o momento de manufatura do produto ou proveniente do seu uso. As modificações no produto buscam alterar a composição, a solidez e os padrões de qualidade do produto e o emprego de produtos substitutos. A reutilização dos resíduos da produção como matéria-prima no processo original ou em outros processos.

Srivastava (2007) analisou que devem ser realizadas mais pesquisas para determinar como as empresas devem selecionar melhor os produtos para cada saída para maximizar o retorno, ao mesmo tempo protegendo a integridade da empresa. Além da Gestão de cadeia de suprimentos verde merece mais atenção e termos de comprometimento de recursos principalmente dentro de uma cadeia de alimentação.

\section{CONSIDERAÇÕES FINAIS}

O presente artigo procurou analisar a tendência da gestão da cadeia de suprimentos verde, envolvendo a produção mais limpa, como práticas de ferramentas ambientais para melhorias dos processos das panificadoras.

A partir de uma revisão bibliográfica da literatura, foi possível apresentar a importância de integrar toda a cadeia do setor para a melhoria ambiental, social e econômica da padaria. Os estudos apresentados mostraram inúmeros benefícios oferecidos pela ferramenta ambiental de produção mais limpa, em consenso com a cadeia de suprimento que trouxe as organizações. 
Quadro 1- Fatores da produção mais limpa que podem acarretar melhorias para a cadeia de suprimento de uma indústria de panificação.

\begin{tabular}{|l|}
\hline $\begin{array}{l}\text { A produção mais limpa traz oportunidade para que a cadeia de suprimento seja gerenciada } \\
\text { de forma sustentável. }\end{array}$ \\
\hline $\begin{array}{l}\text { Ela traz condição para obtenção de melhorias ambientais no processo e desenvolvimento de } \\
\text { produtos, quanto para um mundo mais sustentável. }\end{array}$ \\
\hline Tem importante instrumento para diminuir efeitos que prejudicam o meio ambiente. \\
\hline $\begin{array}{l}\text { Ajuda no gerenciamento de resíduos, práticas de redução deles e a maximização do uso de } \\
\text { recursos naturais e elementos finitos de gestão. }\end{array}$ \\
\hline $\begin{array}{l}\text { Padronizar os insumos e mudanças de matéria-prima, diminuição de resíduos sólidos e da } \\
\text { energia. }\end{array}$ \\
\hline Reduz o lead time, e envolve a incorporação para condutas ambientais. \\
\hline Mesmo existindo algumas barreiras, não é difícil sua implantação nas empresas. \\
\hline $\begin{array}{l}\text { A produção mais limpa ajuda as empresas a ressaltarem o quanto é importante ter uma boa } \\
\text { imagem diante da sociedade por meio de ações de preservação ambiental. }\end{array}$ \\
\hline
\end{tabular}
Fonte: Elaborado pelos autores (2016)

A análise foi feita apenas em uma parte da cadeia de suprimentos, portanto, sugere-se para futuras pesquisas, realizar a análise de toda a cadeia de suprimentos do setor de panificação e analisar como essa prática pode acarretar formas de trazer o setor de panificação uma gestão da cadeia de suprimentos verde eficaz.

\section{REFERÊNCIAS BIBLIOGRÁFICAS}

ABIP, Associação Brasileira da Indústria de Panificação e Confeitaria. Estudo do impacto da inovação tecnológica no setor de panificação e confeitaria. ABIP/ITPC/SEBRAE, 2012. Disponível em:

< http://www.abip.org.br/imagens/file/estudoinovatec9ago12.pdf>. Acesso: 10 de dezembro de 2014.

ABIP, Associação Brasileira da Indústria de Panificação e Confeitaria. Desempenho do Setor de Panificação e Confeitaria Brasileiro de 2012, 2013. Disponível em: < http://www.abip.org.br/perfil_internas.aspx?cod=418>. Acesso em: 10 de dezembro de 2014.

BAAS, L.W. Cleaner Production: beyond projects. Jornal Cleaner Production, n. 3, p. 55-59, 1995.

BARBIERI, J. C. Gestão ambiental empresarial: conceitos, modelos e instrumentos. São Paulo: Saraiva, 2004.

CARDOSO, L. M. F. Indicadores de produção limpa: uma proposta para análise de relatórios ambientais de empresas. Dissertação do Mestrado, Escola Politécnica da Universidade Federal da Bahia, Salvador, 2004. Disponível em: < http://teclim.ufba.br/site/material_online/dissertacoes/dis_ligia_m_f_cardoso.pdf>. Acesso em: 10 de dezembro de 2014.

CNTL, Centro Nacional de Tecnologias Limpas: Implementação de Programas de Produção mais Limpa, 2005. Disponível em: <http//www.cntl.com.br/> . Acesso: 10 de dezembro de 2014. 
DIAS, R. Gestão Ambiental: Responsabilidade social e sustentabilidade. 1.ed.São Paulo: Atlas, 2008.

DIAS, S.L.F.G.; LABEGALINI, L.; CSILLAG, J.M. Sustentabilidade e cadeia de suprimento: uma perspectiva comparada de publicações nacionais e internacionais. Produção, v. 22, n. 3, p. 517-533, 2012.

FERNANDES, L. J. M. ; FREITAS, L.S. Análise do processo produtivo de uma panificadora da cidade de Campina Grande - Paraíba utilizando a ferramenta de P+L. Revista Espacios, v. 35, n. 5, p. 12, 2014.

FERNANDES, S.D.C.; ESPINOSA, J.W.M. Diagnóstico da cadeia de suprimentos no contexto da Produção Mais Limpa: Um estudo de caso da reciclagem de papel. XXX ENEGEP. São Carlos, SP, 2010.

FILHO, J. C. G. S.; SICSÚ, A. B. Produção Mais Limpa: uma ferramenta da Gestão Ambiental aplicada às empesas nacionais. XXIII ENEGEPE. 2003.2 Disponível em: <http://www.abepro.org.br/biblioteca/ENEGEP2003_TR1005_0001.pdf>. Acesso em: 11 de dezembro de 2014.

GLAVIC, P.; LUKMAN, R. Review of sustainability terms and their definitions. Jornal of cleaner production, n. 15, p. 1875-1885, 2007

HENRIQUES, J.; CATARINO, J. Sustainable Value and Cleaner Production e research and application in 19 Portuguese $\mathrm{SME}$, Journal of Cleaner Production. 2014. http://dx.doi.org/10.1016/j.jclepro.2014.02.030. Acesso em: $10 \mathrm{de}$ dezembro de 2014.

HINZ, R. T. P.; VALENTINA, L.V. D; FRANCO, A. C. Sustentabilidade ambiental das organizações através da produção mais limpa ou pela Avaliação do Ciclo de Vida. Estudos Tecnológicos, v. 2, n. 2, p. 91-98. 2006.

JERÔNIMO, C. E. M.; MOURA, T. N.; DANTAS, G. R. Gestão Ambiental em Panificadoras de Mossoró -RN. Rev. Elet. Em Gestão, Educação e Tecnologia Ambiental, v. 10, n. 10, p. 2151-2164, 2013.

MARTINS, G. A.; THEÓPHILO, C. R. Metodologia da Investigação Científica para Ciências Sociais Aplicadas. 2.ed. São Paulo: Editora Atlas S.A, 2009.

PIMENTA, H. C. D.; JÚNIOR, S.M. Modelo de gerenciamento de resíduos sólidos: um estudo de caso na indústria de panificação em Natal - RN. XXVI ENEGEP. Fortaleza, CE, 2006.

PIMENTA, H. C. D. E GOUVINHAS, R. P. Implementação da Produção Mais Limpa na indústria de Panificação de Natal -RN. XXI ENEGEP. Foz do Iguaçu, PR. 2007.

PETTER, R. R.; VAZ, C. R.; RESENDE, L. M. M.; SELIG, P.M. Produção Limpa, Produção mais Limpa, Produção Enxuta, 5s e Manutenção Autônoma - uma proposta metodológica de implantação conjunta. VII Congresso Nacional de Excelência em Gestão, $2011 . \quad$ Disponível em: http://www.excelenciaemgestao.org/portals/2/documents/cneg7/anais/t11_0356_1481.pdf>. Acesso em: 10 de dezembro de 2014.

ROSSI, M. T. B; BARATA, M. M. L. Barreiras à Implementação de Produção Mais Limpa Como Práticas de Ecoeficiência em Pequenas e Médias Empresas no Estado do Rio de Janeiro, 2009. Internacional workshop advances in cleaner production. Disponível em:

<http://www.advancesincleanerproduction.net/second/files/sessoes/4a/1/M.\%20T.\%20B.\%20Rossi\%20\%20Resumo\%20Exp.pdf>. Acesso em: 9 de dezembro de 2014.

SENAI - Produção mais limpa em padarias e confeitarias/senai. Departamento Regional do Rio Grande do Sul. Porto Alegre: $\quad$ SENAl, $2008 . \quad$ Disponível $\quad$ Em: http://wwwapp.sistemafiergs.org.br/portal/page/portal/sfiergs_senai_uos/senairs_uo697/proximos_cursos/Produ \%E7\%E3o\%20mais\%20Limpa\%20em\%20Padarias\%20e\%20Confeitarias.pdf>. Acesso em: 9 de dezembro de 2014.

SEURING, S.; MULLER, M. From a literature review to a conceptual framework for sustainable suplly chain management. Journal of Clean Production. n. 16, p. 1699-1710, 2008.

SCOTTO, G.; CARVALHO, I. C. M.; GUIMARÃES, L. B. Desenvolvimento sustentável. Conceitos fundamentais. Petrópolis/Rio de Janeiro: Vozes, 2007. 
